Tohoku J. Exp. Med., 2003, 200, 145-149

Case Report

\title{
Successful Pregnancy after Myomectomy Using Preoperative Adjuvant Uterine Artery Embolization
}

\author{
Hiroshi Nabeshima, Takashi Murakami, Yumi Sato, Yukihiro Terada, \\ Nobuo Yaegashi and Kunihiro Okamura \\ Department of Obstetrics and Gynecology, Tohoku University School of \\ Medicine, Sendai 980-8574
}

\begin{abstract}
Nabeshima, H., Murakami, T., Sato, Y., Terada, Y., Yaegashi, N. and Okamura, K. Successful Pregnancy after Myomectomy Using Preoperative Adjuvant Uterine Artery Embolization. Tohoku J. Exp. Med., 2003, 200 (3), 145149 _ A 30-year-old-woman presented with uterine leiomyoma and primary sterility. Abdominal myomectomy was performed; however, one cervical leiomyoma was not resected because of a risk of excessive blood loss. Two years after the procedure, a secondary myomectomy using preoperative adjuvant uterine artery embolization (UAE) was performed because of pronounced menorrhagia and her hope for bearing children. The patient's dysmenorrhea disappeared postoperatively and she conceived spontaneously 3 years after the secondary myomectomy. This case suggests that myomectomy using preadjuvant UAE may be an another approach for the treatment of leiomyoma in patients who wish bear children in the future._ leiomyoma; UAE; myomectomy; pregnancy (C) 2003 Tohoku University Medical Press
\end{abstract}

Uterine leiomyoma is the most common benign tumors of the female genital tract. For patients with symptomatic leiomyoma, the choices previously consisted of either medication or surgery, but recently a third choice uterine artery embolization (UAE) has become available (Ravina et al. 1995).

Therefore, patients can now choose medication by the gonadotropin releasing hormone $(\mathrm{GnRH})$ agonist, myomectomy, or UAE. These three treatments have own drawbacks. After $\mathrm{GnRH}$ agonist therapy, leiomyoma often grow larger than before treatment and there are many non-effective cases for $\mathrm{GnRH}$ agonist therapy (Kadowaki et al. 2002). Myomectomy presents technical difficulties, especially in case of huge leiomyomas, diffuse type nodes, cervical leiomyomas, and other types. This treatment carries the potential risk of transfusion due to massive blood loss caused by increased vascularity (Akahira et al. 1998; Haney 2000). UAE has been relatively contraindicated for patients who would like to become pregnant in the future, because of possible complications

Received May 8, 2003; revision accepted for publication July 20, 2003.

Address for reprints: Dr. Hiroshi Nabeshima, Department of Obstetrics and Gynecology, Tohoku

University School of Medicine, 1-1 Seiryomachi, Aoba-ku, Sendai 980-8574, Japan.

e-mail: nabe-akt@umin.ac.jp 
after this treatment, including premature ovarian failure, leiomyoma necrosis, and risk of hysterectomy (Lund et al. 2000).

We report hereby a case of a successful pregnancy after abdominal myomectomy using pre-operative adjuvant UAE for a cervical leiomyoma.

\section{Case Report}

A 30-year-old nulliparous woman underwent treatment for symptomatic leiomyoma. She presented in December 1996 with a threeyear history of severe menorrhagia and primary sterility. GnRH agonist had been administered to this patient at the previous clinic where she was treated, but after resumption of menstruation, the menorrhagia was worse than it had been before treatment.

Transvaginal ultrasonography and magnetic resonance images (MRI) of the pelvic cavity revealed some leiomyomas regions located within the myometrium. A cervical leiomyoma node about $8 \mathrm{~cm}$ in diameter was detected in the posterior area of the uterus
(Fig. 1). Laboratory tests were unremarkable, except for an elevated serum level of CA 125 $(239 \mathrm{U} / \mathrm{ml}$; normal $<35 \mathrm{U} / \mathrm{ml})$. In view of the progressive menorrhagia, an abdominal myomectomy was planned.

Abdominal myomectomy was performed in May 1997. We resected all leiomyoma nodes, except for one cervical node. The cervical node was located in the posterior of the uterus, under the broad ligament. We predicted that the total blood loss during resection would cause a transfusion to be required, or possibly a hysterectomy, and therefore we determined not to remove the cervical node.

Her menorrhagia was milder than before myomectomy, and GnRH agonist was administered post-operatively for 4 months, due to the reduction of residual nodule size. Her menstruation resumed after cessation of medication, and treatments for sterility were performed, including laparoscopy, intra-uterine insemination, and follicle stimulating hormone (FSH) hyper-stimulation. She was given therapies for sterility for two years, but did not conceived.

Her menorrhagia symptoms gradually

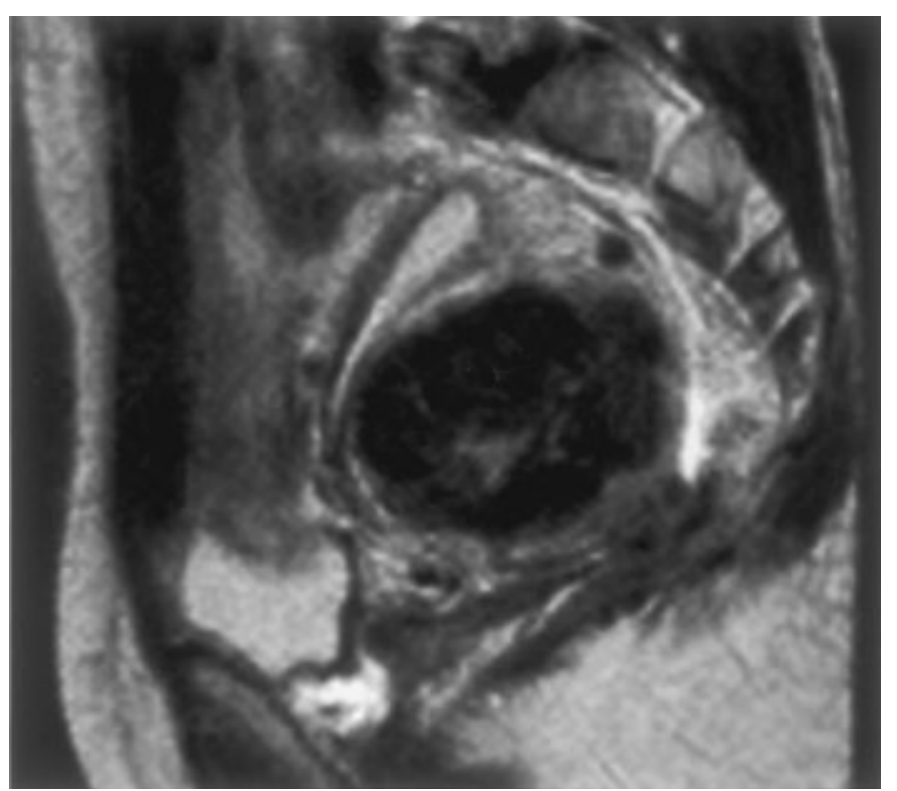

Fig. 1. T2-weighted MR image obtained before myomectomy reveals $8 \mathrm{~cm}$ diameter intramural leiomyoma node at the posterior part of the uterine cervix. 
A

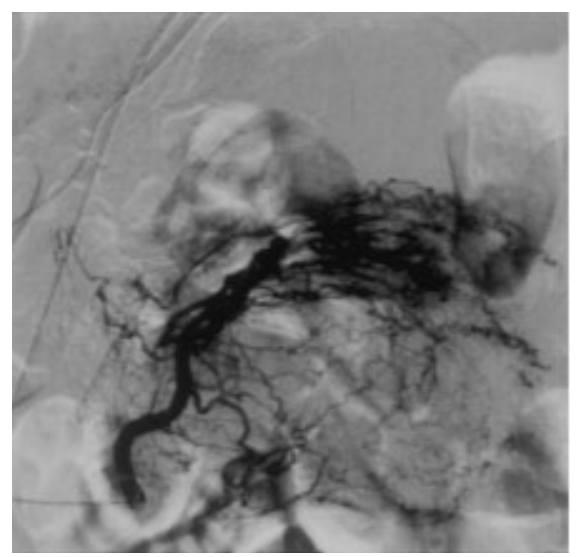

B

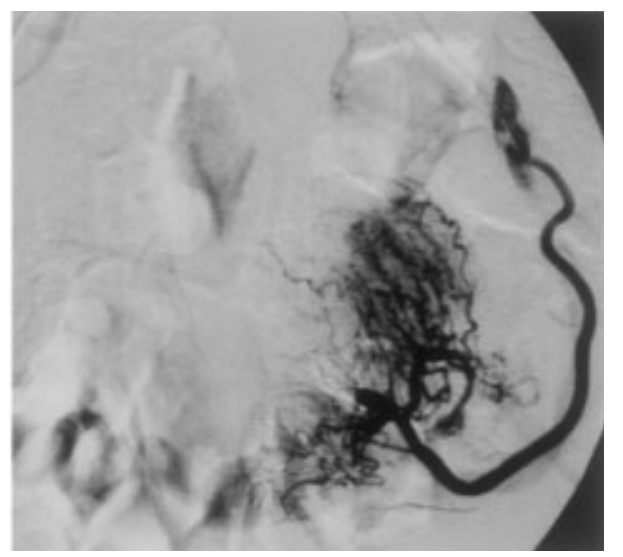

Fig. 2. A: Arteriographic image obtained after selective injection of contrast material into the right uterine artery. B: That of the left uterine artery. Both arteriography images show marked hypervascularity of the myomatous uterus.

appeared during the sterility treatments, and GnRH agonist was administered again for 6 months. After the cessation of GnRH agonist therapy, the symptoms were more pronounced than before treatment, and she had a strong desire to have the cervical node removed. She was informed of the risks of re-myomectomy and UAE, and gave her informed consent to have a myomectomy after UAE to reduce blood loss during surgery.

In December 1999, bilateral uterine artery embolization was performed because of enhanced image of leiomyoma node in arteriography (Fig. 2) using a gelatin sponge (Sponge ${ }^{\circledR}$ ) particles. Four days after the UAE, an abdominal myomectomy was performed. The cervical leiomyoma node was buried under the broad ligament, and was spread over the surrounding tissues diffusely, but the node was completely removed from the myometrium without particular problems during the operation. The total operating time was 136 minutes, the blood loss was $60 \mathrm{ml}$, and the post-operative course was uneventful.

One month later, her menstruation resumed without severe menorrhagia. Treatments for sterility were carried out from 6 months after myomectomy. In May 2002, she conceived following a natural menstrual cycle. The pregnancy was uneventful, and elective cesarean section was performed with term pregnancy in January 2003.

\section{DISCUSSION}

To reduce surgical blood loss, the intra myometrial injection of vasopressin is used routinely in our hospital. However, there are still some cases where transfusion is required, especially when the uterus contains a cervical leiomyoma and/or many multiple nodes.

We have been attempting a new preliminary treatment of pre-operative adjuvant $\mathrm{UAE}$ using a gelatin sponge to overcome the problems that occur with a difficult type of myomectomy. In preliminary treatments, there have been no cases in which a transfusion or hysterectomy was required, and no cases needing additional therapy have yet occurred. However, we stopped performing this procedure in patients who would like to bear children in the future, because of risk of premature ovarian failure (Bradley et al. 1998), hysterectomy (Goodwin et al. 1997), uterine rupture (Shashoua et al. 2002), and death after UAE (Vashisht et al. 1999).

There are few reports that describe surgi- 
cal adjuvant UAE. Goodwin et al. (1998) reported that UAE has been proposed as a surgical adjuvant before myomectomy to reduce intraoperative bleeding. And in preliminary cases of myomectomy with pre-operative adjuvant UAE of our hospital, the procedure is effective in reducing operative blood loss. However, Hurst et al. (2000) mentioned that routine treatment by embolization before surgery is not encouraged, because there are no studies to support the safety of this approach. Although we agree with this opinion, we think that preoperative adjuvant UAE may be justified in special cases like the one described in this report because of the life threatened risk of myomectomy.

Preoperative adjuvant UAE is an expensive therapy. Recently, there have been some reports of pregnancy after UAE (Ravina et al. 2000; McLucas et al. 2001; Vashisht et al. 2001), so it may be possible to perform UAE in cases like the one we treated. Which treatment is optimal in contributing to fertility needs to be studied in the future. Until we treated this patient, there had been no reports of pregnancy after UAE, and therefore we chose myomectomy after UAE, for the reason mentioned previously.

In conclusion, we demonstrated a case of a successful pregnancy after myomectomy using preoperative adjuvant UAE. Myomectomy using pre-operative adjuvant UAE appears to be an another approach for difficult type of leiomyoma, because it may reduce blood loss, and enable pregnancy to occur, as in our case. However, the outcome of pregnancy following this procedure is not established, and therefore we believe that further investigation is needed.

\section{References}

Akahira, J., Ito, K., Forman, R.G., Nakamura, R. \& Yajima, A. (1998) Massive intraperitoneal hemorrhage and hypovolemic shock due to rupture of a coronary vessel of a uterine leiomyoma: a report of two cases. Tohoku J.
Exp. Med., 185, 217-222.

Bradley, E.A., Reidy, J.F., Forman, R.G., Jarosz, J. \& Braude, P.R. (1998) Transcatheter uterine artery embolisation to treat large uterine fibroids. Br. J. Obstet. Gynaecol., 105, 235-240.

Haney, A.F. (2000) Clinical decision making regarding leiomyomata: What we need in the next millenium. Environ. Health Perspect., Suppl. 5, 108, 835-839.

Hurst, B.S., Stackhouse, D.J., Matthews, M.L. \& Marshburn, P.B. (2000) Uterine artery embolization for symptomatic uterine myomas. Fertil. Steril., 74, 855-869.

Lund, N., Justesen, P., Elle, B., Thomsen, S.G. \& Floridon, C. (2000) Fibroids treated by uterine artery embolization: A review. Acta Obstet. Gynecol. Scand., 79, 905-910.

McLucas, B., Goodwin, S., Adler, L., Rappaport, A., Reed, R. \& Perrella, R. (2001) Pregnancy following uterine fibroid embolization. Int. J. Gynaecol. Obstet., 74, 1-7.

Goodwin, S.C., Vedantham, S., McLucas, B., Forno, A.E. \& Perrella, R. (1997) Preliminary experience with uterine artery embolization for uterine fibroids. J. Vasc. Interv. Radiol., 8, 517-526.

Goodwin, S.C. \& Walker, W.J. (1998) Uterine artery embolization for the treatment of uterine fibroids. Curr. Opin. Obstet. Gynecol., 10, 315-320.

Kadowaki, M., Murakami, T., Morita, J., Terada, Y., Yaegashi, N. \& Okamura, K. (2002) Prediction of the effects of gonadotropin-releasing hormone agonist therapy in uterine leiomyoma by $\mathrm{T} 1$ contrast-enhanced magnetic resonance imaging sequences. Fertil. Steril., 77, 1081-1082.

Ravina, J.H., Herbreteau, D., Ciraru-Vigneron, N., Bouret, J.M., Houdart, E., Aymard, A. \& Merland, J.J. (1995) Arterial embolisation to treat uterine myomata. Lancet, 346, 671-672.

Ravina, J.H., Vigneron, N.C., Aymard ALe Dref, O. \& Merland, J.J. (2000) Pregnancy after embolization of uterine myoma: Report of 12 cases. Fertil. Steril., 73, 1241-1243.

Shashoua, A.R., Stringer, N.H., Pearlman, J.B., Behmaram, B. \& Stringer, E.A. (2002) Ischemic uterine rupture and hysterectomy 3 months after uterine artery embolization. J. Am. Assoc. Gynecol. Laparosc., 9, 217-220.

Vashisht, A., Studd, J., Carey, A. \& Burn, P. (1999) Fatal septicaemia after fibroid embolisation. 
Lancet, 354, 307-308.

Vashisht, A., Smith, J.R., Thorpe-Beeston, G. \& McCall, J. (2001) Pregnancy subsequent to uterine artery embolization. Fertil. Steril., 75, 1246-1248. 